\title{
Dioscoreaceae endémicas del Perú
}

\section{Blanca León ${ }^{1,2}$}

${ }^{1}$ Museo de Historia Natural, Av. Arenales 1256, Aptdo. 14-0434, Lima 14, Perú

2 Plant Resources Center, University of Texas at Austin, Austin TX 78712 EE.UU.

blanca.leon@mail.utexas.edu

\section{Resumen}

La familia Dioscoreaceae está conformada por bejucos y hierbas. Para la flora peruana, el género, Dioscorea incluye 77 especies (Brako \& Zarucchi, 1993; Ulloa Ulloa et al., 2004). En este trabajo se reconoce 21 endemismos. Se asignó las categorías de amenaza de la UICN a cinco especies. Los taxones endémicos de esta familia ocupan un amplio rango de regiones ecológicas que van de los 100 a $4250 \mathrm{~m}$ de altitud, desde el Desierto Subtropical Costero, Matorral Desértico hasta la Puna Húmeda y Seca y Páramo; sin embargo, la mayoría de los endemismos se hallan en la región del Bosque Húmedo Montano. Seis de las especies endémicas se encuentran representadas en áreas naturales protegidas.

Palabras claves: Dioscoreaceae, Dioscorea, Perú, endemismo, plantas endémicas.

\section{Abstract}

The Dioscoreaceae family consists of vines and herbs. In the Peruvian flora, the family is represented by 77 species in the genus Dioscorea (Brako \& Zarucchi, 1993; Ulloa Ulloa et al., 2004). Here we recognize 21 endemic taxa. We assigned IUCN threat categories to five species. The endemic taxa in this family occupy a wide range of ecological regions between 100 and 4250 m elevation, from the Subtropical Coastal Desert to the Mesic Shrubland and Dry and Humid Puna and Paramo; however, the majority of endemics are found in the Humid Montane Forest region. Six species endemic to Peru have been reported to occur in the country's protected areas network.

Keywords: Dioscoreaceae, Dioscorea, Peru, endemism, endemic plants.

\section{Dioscorea ainensis R. Knuth}

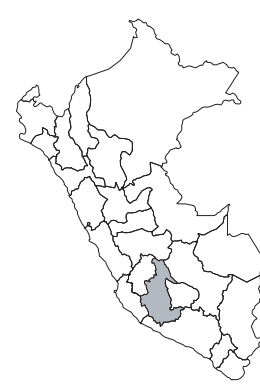

Publicación: Repert. Spec. Nov. Regni Veg. 29: 93. 1931.

Colección tipo: E.P. Killip \& A.C. Smith 22742

Herbarios: B, F, NY, US.

Nombre común: Desconocido.

Registro departamental: AY.

Regiones Ecológicas: BMHP; 750—1000

$\mathrm{m}$.

SINANPE: Sin registro.

Herbarios peruanos: Ninguno.

Observaciones: Este taxón fue considerado por Brako \& Zarucchi (1993) como un endemismo; sin embargo, no ha sido posible evaluarlo, ni asignarle una categoría.

\section{Dioscorea ancachsensis R. Knuth}

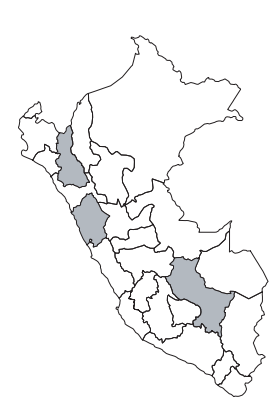

\section{VU, B1a}

Publicación: Pflanzenr. 4(43): 202. 1924. Colección tipo: A. Weberbauer 7249

Herbarios: B, MO, NY; AMAZ.

Nombre común: Desconocido.

Registro departamental: AN, CA, CU. Regiones Ecológicas: PSH, PAR; 3000_ $4250 \mathrm{~m}$.

SINANPE: PNH

Herbarios peruanos: AMAZ (fragmento tipo).

Observaciones: Esta especie herbácea se conoce de varias colecciones, realizadas durante los últimos 25 años. Las localidades más extremas distan más de $1020 \mathrm{~km}$. Habita ambientes rocosos de la puna y páramo, ambas zonas naturalmente fragmentadas, reciben impacto de quema y ganadería.

\section{Dioscorea andromedusae O. Téllez}

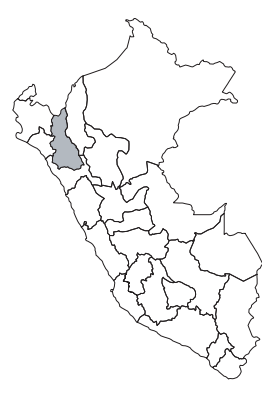

\section{EN, B1ab(iii)}

Publicación: Brittonia 48(1): 103, f. 2. 1996.

Colección tipo: J. Mostacero L. et al. 1691 Herbarios: MEXU, MO; HUT.

Nombre común: Desconocido.

Registro departamental: CA.

Regiones Ecológicas: BMHM; $2460 \mathrm{~m}$. SINANPE: PNC

Herbarios peruanos: HUT (isotipo citado).

Observaciones: Bejuco conocido de pocas localidades en el Parque Nacional Cutervo. Fue descrita de una planta recolectada en 1986. Amenazas a sus poblaciones podrían estar asociadas a la deforestación.

\section{Dioscorea callacatensis R. Knuth}

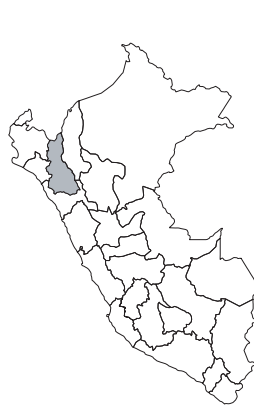

\section{DD}

Publicación: Repert. Spec. Nov. Regni Veg. 28: 83. 1930.

Colección tipo: A. Raimondi 5631

Herbarios: B.

Nombre común: Desconocido.

Registro departamental: CA.

Regiones Ecológicas: Sin datos; altitud desconocida.

SINANPE: Sin registro.

Herbarios peruanos: Ninguno.

Observaciones: Bejuco conocido de la colección tipo, una planta recolectada en la cuenca del Chotano (Hensold, 1999). Al parecer, no ha sido registrado desde 1879 . 


\section{Dioscorea chagllaensis R. Knuth}

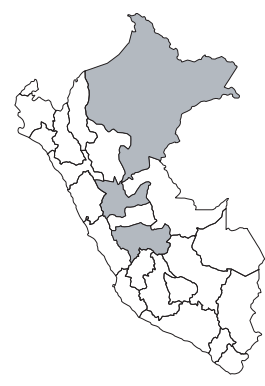

Publicación: Repert. Spec. Nov. Regni Veg. 28: 88. 1930.

Colección tipo: J.F. Macbride 3651

Herbarios: F, US.

Nombre común: Desconocido.

Registro departamental: HU, JU, LO

Regiones Ecológicas: BMHM; 2400$800 \mathrm{~m}$.

SINANPE: Sin registro

Herbarios peruanos: USM (1)?

Observaciones: Esta especie no fue considerada por Brako \& Zarucchi (1993) como un endemismo. El ejemplar tipo en el herbario US, cita erróneamente la localidad en Cusco, siguiendo a Ayala (1998), pero corresponde a Huánuco. No ha sido posible evaluarla, ni asignarle una categoría.

\section{Dioscorea chancayensis R. Knuth}

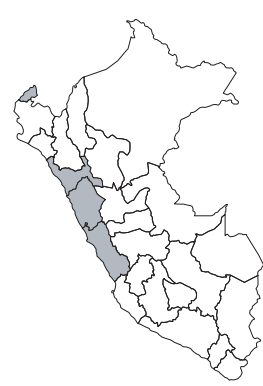

\section{NT}

Publicación: Repert. Spec. Nov. Regni Veg. 28: 81—82. 1930.

Colección tipo: A. Weberbauer 7486

Herbarios: F.

Nombre común: Desconocido.

Registro departamental: AN, LI, LL, TU. Regiones Ecológicas: DCT, DST; 200$400 \mathrm{~m}$.

SINANPE: RNL

Herbarios peruanos: HUT (1), USM (5).

Observaciones: Hierba escandente, conocida de localidades naturalmente fragmentadas, en el norte y centro de la vertiente del Pacífico. Esta especie no fue incluida por Brako \& Zarucchi (1993) como endémica. Con excepción de una colección, todos los ejemplares fueron recolectados en ambientes de lomas.

\section{Dioscorea chaponensis R. Knuth var. chazutensis F. Ayala}

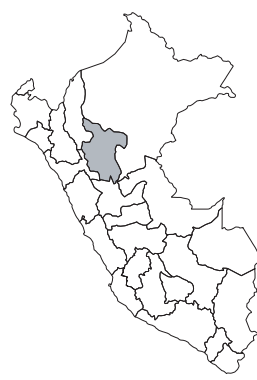

Publicación: Dioscoreaceae Perú, p. 22, f. 8. 1998.

Colección tipo: G. Klug 4133

Herbarios: F, GH, MO, US.

Nombre común: Desconocido.

Registro departamental: SM.

Regiones Ecológicas: BHA; $260 \mathrm{~m}$.

SINANPE: Sin registro.

Herbarios peruanos: Ninguno.

Observaciones: Este taxón se conoce solamente de una localidad, recolectado en 1935, en la cuenca del Huallaga. No ha sido posible evaluarlo, ni asignarle una categoría.

\section{Dioscorea ferreyrae F. Ayala}

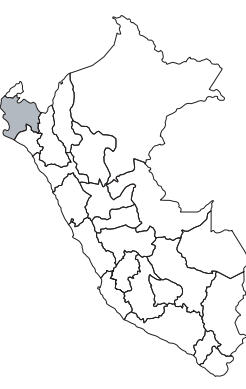

Publicación: Dioscoreaceae Perú, p. 27. 1998. Colección tipo: R. Ferreyra 10892

Herbarios: US; AMAZ, USM.

Nombre común: Desconocido.

Registro departamental: PI.

Regiones Ecológicas: MDE; 1400—1500 m.

SINANPE: Sin registro.

Herbarios peruanos: AMAZ (isotipo citado), USM (isotipo citado).
Observaciones: Este taxón fue descrito posterior a Brako \& Zarucchi (1993); no ha sido posible evaluarlo, ni asignarle una categoría.

\section{Dioscorea gentryi O. Téllez}

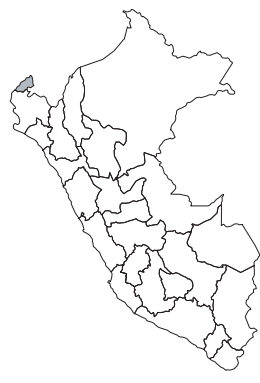

Publicación: Brittonia 48(1): 100, 103, f. 1. 1996.

Colección tipo: A.H. Gentry \& C. Diaz 58260

Herbarios: MEXU, MO; $\underline{\mathrm{HUT}}$.

Nombre común: Desconocido.

Registro departamental: TU.

Regiones Ecológicas: MDE; $820 — 850 \mathrm{~m}$.

SINANPE: PNCAm

Herbarios peruanos: HUT (isotipo citado).

Observaciones: Hierba voluble conocida solamente del norte del país. No ha sido posible evaluarla, ni asignarle una categoría.

\section{Dioscorea haenkeana C. Pres1}

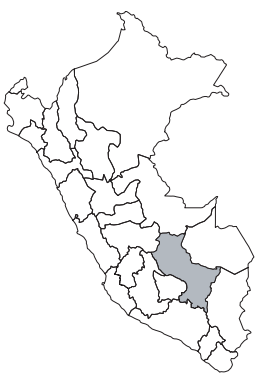

Publicación: Reliq. Haenk. 1(2): 1135. 1827. Colección tipo: T. Haenke s.n.

Herbarios: B.

Nombre común: Desconocido.

Registro departamental: CU.

Regiones Ecológicas: $\mathrm{BHA}$; altitud desconocida.

SINANPE: Sin registro.

Herbarios peruanos: CUZ (1)?.

Observaciones: Este taxón fue considerado por Brako \& Zarucchi (1993) como un endemismo; sin embargo, no ha sido posible evaluarlo, ni asignarle una categoría.

\section{Dioscorea huallagensis R. Knuth}

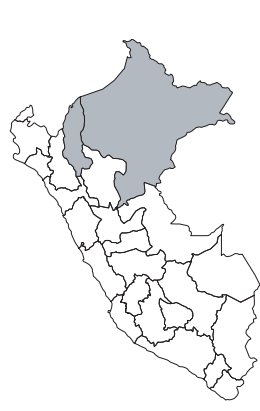

\section{EN, B1ab(iii)}

Publicación: Repert. Spec. Nov. Regni Veg. 29: 95-96. 1931.

Colección tipo: E.P. Killip \& A.C. Smith 27660

Herbarios: B, NY.

Nombre común: Desconocido.

Registro departamental: AM, LO.

Regiones Ecológicas: BMHP, BHA; 135-1700 m.

SINANPE: ZRCC

Herbarios peruanos: AMAZ (1).

Observaciones: Bejuco conocido de unas pocas localidades dispersas en la Amazonía peruana. Fue descrita de una localidad en la cuenca del Huallaga. Una población se halla en la Zona Reservada Cordillera Colán. La deforestación es su principal amenaza.

\section{Dioscorea incayensis R. Knuth}

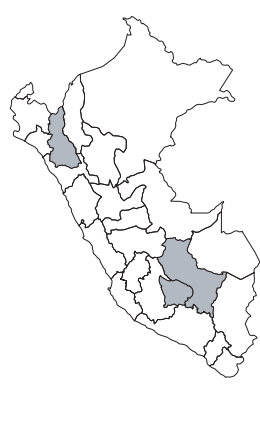

Publicación: Repert. Spec. Nov. Regni Veg. 28: 85. 1930.

Colección tipo: F. Herrera 2300

Herbarios: B.

Nombre común: Desconocido.

Registro departamental: AP, CA, CU.

Regiones Ecológicas: BMHM; 2300$2950 \mathrm{~m}$.

SINANPE: SHMP

Herbarios peruanos: CUZ (3). 
Observaciones: Este taxón fue considerado por Brako \& Zarucchi (1993) como un endemismo; sin embargo, no ha sido posible evaluarlo, ni asignarle una categoría.

\section{Dioscorea macbrideana R. Knuth}

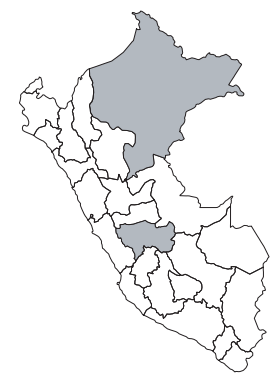

Publicación: Repert. Spec. Nov. Regni Veg. 28: 87-88. 1930.

Colección tipo: J.F. Macbride 5347

Herbarios: F.

Nombre común: Desconocido.

Registro departamental: JU, LO.

Regiones Ecológicas: BMHP, BHA; 100-800 m.

SINANPE: RNAM

Herbarios peruanos: Ninguno.

Observaciones: Este taxón fue considerado por Brako \& Zarucchi (1993) como un endemismo; sin embargo, no ha sido posible evaluarlo, ni asignarle una categoría.

\section{Dioscorea monadelphoides J.F. Macbr. var. longirachis (R. Knuth) Ayala}

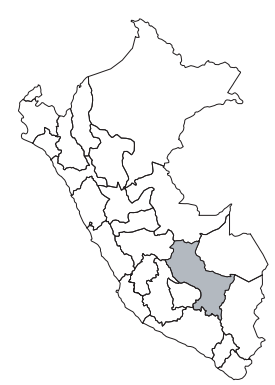

Publicación: Dioscoreaceae Perú, p. 38. 1998. Colección tipo: A. Weberbauer 7862

Herbarios: B, F, US.

Nombre común: Desconocido.

Registro departamental: CU.

Regiones Ecológicas: MA, BMHM; 1300-2850 m.

SINANPE: Sin registro.

Herbarios peruanos: Ninguno.

Observaciones: Este taxón fue considerado por Brako \& Zarucchi (1993) como un endemismo a nivel de especie; sin embargo, no ha sido posible evaluarlo, ni asignarle una categoría.

\section{Dioscorea poeppigii Kunth}

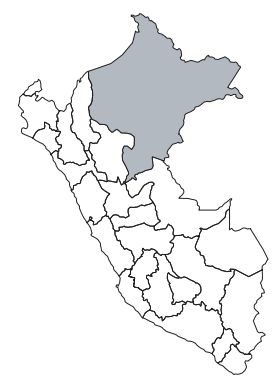

Publicación: Enum. Pl. 5: 365. 1850.

Colección tipo: E.F. Poeppig 2356

Herbarios: B.

Nombre común: Desconocido.

Registro departamental: LO.

Regiones Ecológicas: Sin datos; altitud desconocida.

SINANPE: Sin registro.

Herbarios peruanos: Ninguno.

Observaciones: Este taxón fue considerado por Brako \& Zarucchi (1993) como un endemismo; sin embargo, no ha sido posible evaluarlo, ni asignarle una categoría.

\section{Dioscorea putisensis R. Knuth}

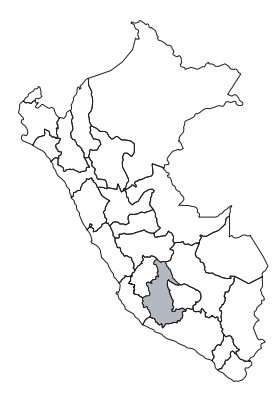

Publicación: Repert. Spec. Nov. Regni Veg. 28: 82. 1930.

Colección tipo: A. Weberbauer 7534

Herbarios: F.

Nombre común: Desconocido.

Registro departamental: AY.

Regiones Ecológicas: BPM; $3200 \mathrm{~m}$.

SINANPE: Sin registro.

Herbarios peruanos: Ninguno.
Observaciones: Esta especie no fue considerada en Brako \& Zarucchi (1993) como endémica. No ha sido posible evaluarla, ni asignarle una categoría.

\section{Dioscorea quispicanchensis R. Knuth}

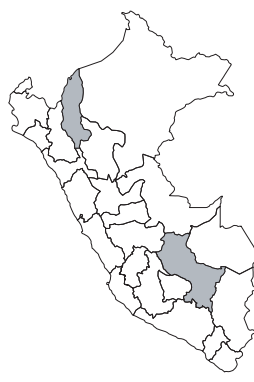

Publicación: Repert. Spec. Nov. Regni Veg. 29: 93. 1931.

Colección tipo: A. Weberbauer 7810

Herbarios: B.

Nombre común: Desconocido.

Registro departamental: AM, CU.

Regiones Ecológicas: BMHM; 1200$1400 \mathrm{~m}$

SINANPE: Sin registro.

Herbarios peruanos: Ninguno.

Observaciones: Este bejuco aparentemente se conoce de dos localidades disyuntas en la vertiente oriental. El ejemplar tipo fue recolectado en 1929 de la cuenca del Marcapata. No ha sido posible evaluarlo, ni asignarle una categoría.

\section{Dioscorea sandiensis R. Knuth}

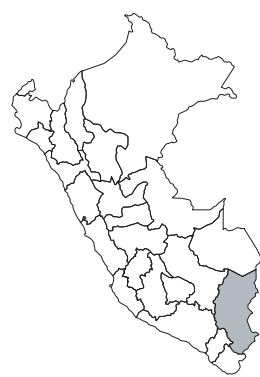

Publicación: Notizbl. Bot. Gart. BerlinDahlem 7(65): 192-193. 1917.

Colección tipo: A. Weberbauer 668

Herbarios: B.

Nombre común: Desconocido.

Registro departamental: PU.

Regiones Ecológicas: BMHM; altitud desconocida.

SINANPE: Sin registro.

Herbarios peruanos: Ninguno.

Observaciones: Este taxón fue considerado por Brako \& Zarucchi (1993) como un endemismo; sin embargo, no ha sido posible evaluarlo, ni asignarle una categoría.

\section{Dioscorea schubertiae Ayala}

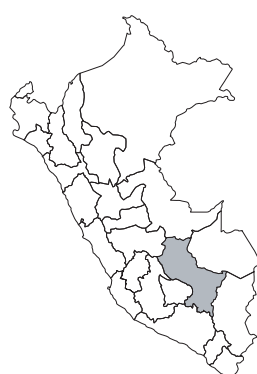

Publicación: Phytologia 55(5): 296. 1984. Colección tipo: A. Weberbauer 7815

Herbarios: F, US.

Nombre común: Desconocido.

Registro departamental: CU.

Regiones Ecológicas: BMHM; $2800 \mathrm{~m}$.

SINANPE: Sin registro.

Herbarios peruanos: Ninguno.

Observaciones: Este taxón fue considerado por Brako \& Zarucchi (1993) como un endemismo; sin embargo, no ha sido posible evaluarlo, ni asignarle una categoría.

\section{Dioscorea tambillensis R. Knuth}

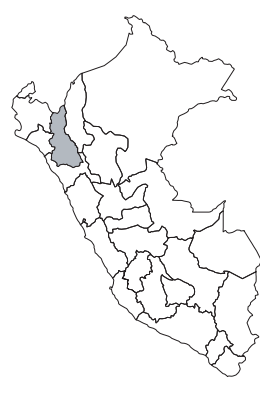

Publicación: Repert. Spec. Nov. Regni Veg. 28: 81. 1930.

Colección tipo: A. Raimondi 3346

Herbarios: B.

Nombre común: Desconocido.

Registro departamental: CA.

Regiones Ecológicas: BMHM; altitud desconocida.

SINANPE: Sin registro.

Herbarios peruanos: Ninguno. 
Observaciones: Este taxón fue considerado por Brako \& Zarucchi (1993) como un endemismo; sin embargo, no ha sido posible evaluarlo, ni asignarle una categoría.

\section{Dioscorea vargasii Standl.}

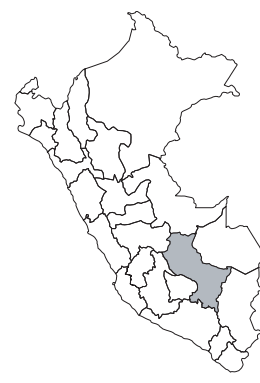

Publicación: Publ. Field Mus. Nat. Hist., Bot. Ser. 22(3): 134. 1940.

Colección tipo: C. Vargas C. 691

Herbarios: F.

Nombre común: Desconocido.

Registro departamental: CU.

Regiones Ecológicas: MA; $2960 \mathrm{~m}$.

SINANPE: Sin registro.

Herbarios peruanos: Ninguno.

Observaciones: Este taxón fue considerado por Brako \& Zarucchi (1993) como un endemismo; sin embargo, no ha sido posible evaluarlo, ni asignarle una categoría. 